\title{
A CHANGE OF RINGS THEOREM AND THE ARTIN-REES PROPERTY
}

\author{
M. BORATYŃSKI
}

ABSTRACT. A two-sided ideal 2 of the ring $R$ is said to have the left $A R$ property if for every left ideal $I$ and every $k$ there exists an $n$ such that $\vartheta^{n} \cap I \subset \mathscr{U}^{k} I$. Let $R$ be a left noetherian ring and $थ$ a two-sided ideal contained in its Jacobson radical. If $\mathscr{U}$ has the $\mathrm{AR}$ property then $1 \mathrm{gl} \operatorname{dim} R \leq$ $\mathrm{p} \operatorname{dim} R / \imath+1 \mathrm{gl} \operatorname{dim} R / \imath$, where $\mathrm{p} \operatorname{dim} R /$ थ denotes the (left) projective dimension of the module $R /$ r.

Let $R$ be a left noetherian ring and $J$ its Jacobson radical. Small has proved in [7] that $1 \mathrm{gl} \operatorname{dim} R \leq \mathrm{r} w \operatorname{dim} R / \mathscr{U}+1 \mathrm{gl} \operatorname{dim} R / \mathscr{U}$, where $\mathscr{Q}$ is a twosided ideal contained in $J$. The natural question arises whether in the above formula right weak dimension can be replaced by left projective dimension. Fields [4] has produced an example which shows that the answer, in general, is "no". However we are able to prove that in case $\mathfrak{U}$ satisfies the AR property the answer is "yes".

Our result is derived from three lemmas. The first is a homological characterization of the AR property which may be of some independent interest and the third is an improvement of Small's result.

Definition. A two-sided ideal $\mathfrak{U}$ of the ring $R$ is said to have the (left) AR property if for every left ideal $I$ and every $k$ there exists an $n$ such that $\mathfrak{U}^{n} \cap I \subset \mathfrak{U}^{k} I$.

Remark. In the commutative noetherian case it follows from the ArtinRees lemma that every ideal has the AR property.

In the sequel, $E(M)$ will denote an injective hull of the left module $M$.

Lemma 1. Let $R$ be a left noetherian ring and $\mathcal{U}$ its arbitrary two-sided ideal. Then the following two conditions are equivalent.

1. I has the AR property.

$2^{\circ}$. $E(M)=\bigcup_{i}\left(0: 2 Z^{i}\right)$ for any left module $M$ whose every element is annihilated by some power of $\mathfrak{U}$ where $\left(0: \mathfrak{U}^{i}\right)=\left\{x \in E(M) \mid \mathfrak{\mathfrak { U } ^ { i } x}=0\right\}$.

Proof. $1^{\circ} \rightarrow 2^{\circ}$. We put $E_{1}=\bigcup_{i}\left(0: \mathfrak{U}^{i}\right)$. It will be sufficient to prove

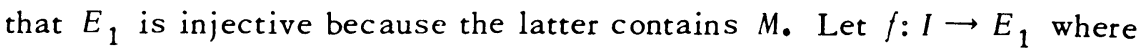

Received by the editors September 19, 1974 and, in revised form, October 7, 1974.

AMS (MOS) subject classifications (1970). Primary 16A60.

Key words and phrases. AR property, projective dimension, Jacobson radical. 
$I$ is an arbitrary left ideal of the ring $R . f(I) \subset\left(0: \gtrless^{k}\right)$ for some $k$ because $I$ is finitely generated. We pick $n$ such that $\mathfrak{U}^{n} \cap I \subset \mathfrak{Q}^{k} I$. $\mathfrak{U}^{n} \cap I$ is contained in the kernel of $f$ because $f\left(\mathfrak{y}^{n} \cap I\right) \subset f\left(2 \mathfrak{l}^{k} I\right)=: \mathfrak{Q}^{k} f(I)=0$.

We have the diagram of $R / \mathfrak{Q}^{n}$-modules with an exact row:

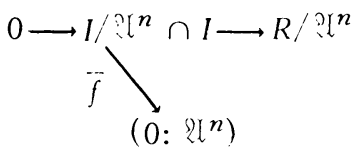

$\bar{f}$ is the homomorphism induced by $f$ (we can assume that $n>k)$. (0: $\left.\mathfrak{U}^{n}\right)$ is an injective $R / \mathfrak{Q}^{n}$-module. So there exists a homomorphism $R / \mathfrak{R}^{n} \rightarrow\left(0: \mathfrak{Q}^{n}\right)$ which makes the above diagram commutative. This homomorphism composed with the natural homomorphism $R \rightarrow R / \mathfrak{Q t}^{n}$ is the desired extension of $f$ to $R$.

$2^{\circ} \rightarrow 1^{\circ}$. Let us consider the natural homomorphism $f: I \rightarrow I / \mathfrak{Q} \mathfrak{L}^{k} I C$ $E\left(I / \mathscr{U}^{k} I\right)$. There exists an element $x \in E\left(I / \mathscr{U}^{k} I\right)$ such that $f(r)=r x$ for all $r \in I$. By our assumption $x \in\left(0: 2 \mathfrak{l}^{n}\right)$ for some $n$. It follows that $2 \mathfrak{l}^{n} \cap I$ is contained in the kernel of $f$, which coincides with $\mathfrak{l}^{k} I$, which was to be proved. Several cases of the above lemma are known [3], [5], [6].

In the sequel inj $\operatorname{dim} M, \mathrm{p} \operatorname{dim} M$ will denote the injective and the projective dimension of the left module $M$ respectively.

Lemma 2. Let $R$ be a left noctherian ring and $2 \mathrm{l}$ its tuo-sided ideal which has the $A R$ property. If $M$ is a module whose every element is annihilated by some power of 2t then $i n j \operatorname{dim} M \leq \sup \mathrm{p} \operatorname{dim} S$, where supremum is taken over the category of module's which are annibilated by $\mathfrak{A}$.

Proof. It will suffice to prove that if $\operatorname{Ext}^{n+1}(S, M)=0$ for all modules $S$ which are annihilated by $\mathfrak{l}$, then $\operatorname{inj} \operatorname{dim} M \leq n$. We start with $n=0$. If $E(M) / M \neq 0$, then, by Lemma 1 , this module contains a nonzero submodule $M_{1} / M$ which is annihilated by 2 . We have the exact sequence $0 \rightarrow M \rightarrow$ $M_{1} \rightarrow M_{1} / M \rightarrow 0$ which is nonsplitting because $E(M)$ is an essential extension of $M$ (i.e. every nonzero submodule of $E(M)$ has a nonzero intersection with $M)$. This exact sequence represents a nonzero element of $\operatorname{Ext}^{1}\left(M_{1} / M, M\right)$ which contradicts our hypothesis.

Let $n: 0$. We consider the exact sequence $0 \rightarrow M \rightarrow E(M) \rightarrow E(M) / M$ $\rightarrow 0, \operatorname{Ext}^{n}(S, E(M), M) \cdots \operatorname{Ext}^{n+1}(S, M)=0$. By Lemma 1, each element of $E(M) / M$ is annihilated by some power of 2 . So by the inductive hypothesis, inj $\operatorname{dim} F(M) / M \leq n-1$. We infer that inj $\operatorname{dim} M \leq n$, which was to be proved.

I.emma 3. Let $R$ be a left noetherian ring and $J$ its Jacobson radical. Then $1 \mathrm{gl} \operatorname{dim} R=\sup i n j \operatorname{dim} S$, uhere supremum is taken ower the category of modules uhich are annibilated by $J$.

Proof. Let $M$ be a finitely generated module. We shall prove that the condition $\operatorname{Ext}^{n+1}(M, S) \ldots 0$ for all modules $S$ which are annihilated by $J$ 
implies that $\mathrm{p} \operatorname{dim} M \leq n$. Let $n=0$. We have an exact sequence $0 \rightarrow K$ $\stackrel{a}{\rightarrow} F \rightarrow M \rightarrow 0$, where $F$ is a finitely generated free module. The map induced by $a, \operatorname{Hom}(F, K / J K) \rightarrow \operatorname{Hom}(K, K / J K)$, is an epimorphism because $\operatorname{Ext}^{1}(M, K / J K)=0$. In particular the natural homomorphism $K \rightarrow K / J K$ which will be denoted by $\gamma$ can be extended to the homomorphism $\delta: F \rightarrow K / J K$. $F$ is a free module, so there exist $\beta: F \rightarrow K$ such that $\gamma \beta=\delta$. We obtain that $\gamma \beta \alpha=\gamma$. Let us put $\epsilon=\beta \alpha-1$. Then $\epsilon: K \rightarrow K$ and $\epsilon(K) \subset J K$. By Nakayama's lemma, the homomorphism $1+\epsilon$ is an epimorphism. It follows that $1+\epsilon$ is also a monomorphism [1, Chapter $8, \mathrm{p} .23]$ because $K$ is a noetherian module. So there exists $\beta^{\prime}: K \rightarrow K$ such that $\beta^{\prime}(1+\epsilon)=\beta^{\prime} \beta a=1$. We have obtained that the exact sequence $0 \rightarrow K \stackrel{a}{\rightarrow} F \rightarrow M \rightarrow 0$ is splitting and $M$ is a projective module. The induction step will be omitted because of its routineness.

It suffices to prove the inequality $1 \mathrm{gl} \operatorname{dim} R \leq \sup$ inj $\operatorname{dim} S$. If the right side of this formula is infinite there is nothing to do. Let $\sup$ inj $\operatorname{dim} S=$ $n<\propto$ and $M$ be an arbitrary finitely generated module. Then $\operatorname{Ext}^{n+1}(M, S)$ $=0$ for all modules $S$ which are annihilated by $J$. We obtain that $\mathrm{p} \operatorname{dim} M$ $\leq n$. So $1 \mathrm{gl} \operatorname{dim} R \leq n$ which was to be proved.

Remark. Small's result mentioned at the beginning of our paper easily follows from the above lemma. It suffices to use the formula

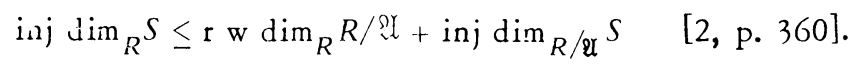

Theorem. Let $R$ be a left noetherian ring and $\mathbb{U}$ a two-sided ideal contained in its Jacobson radical. If $\mathcal{U}$ has the AR property, then

$$
1 \mathrm{gl} \operatorname{din} R \leq p \operatorname{dim} R / \mathfrak{l}+1 \mathrm{gl} \operatorname{dim} R / \mathfrak{l}
$$

Proof. By Lemma 3, $1 \mathrm{gl} \operatorname{dim} R=\sup i n j \operatorname{dim} S$, where supremum is taken over the category of modules which are annilated by 2 . We infer from Lemma 2 that $1 \mathrm{gl} \operatorname{dim} R \leq \sup \mathrm{p} \operatorname{dim} S$, where supremum is taken over the above mentioned category. Our assertion will now follow from the formula

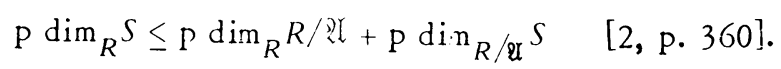

Corollary. Let $R$ be a left noetherian semilocal ring and $J$ its Jacobson radical. If $J$ has the $A R$ property, then $1 \mathrm{gl} \operatorname{dim} R=\mathrm{p} \operatorname{dim} R / J$.

In view of the above result one would like to mention the following open problem: Is it true that the Jacobson radical of every left and right noetherian ring $R$ has the left AR property? In this direction the author was able to prove that it is so in case $R$ is, moreover, semilocal and has the property that all its left ideals are two-sided.

I would like to express my thanks to the reviewer for his helpful remarks. 


\section{REFERENCES}

1. N. Bourbaki, Éléments de mathématique. XXIII. Part 1. Les structures fondamentales de l'analyse. Livre II: Algèbre. Chap. 8, Actualités Sci. Indust., no. 1261, Hermann, Paris, 1958. MR 20 \#4576.

2. H. Cartan and S. Eilenberg, Homological algebra, Princeton Univ. Press, Princeton, N. J., 1956. MR 17, 1040.

3. J. C. McConnell, Localisation in enveloping rings, J. London Math. Soc. 43 (1968), 421-428. MR 37 \#4112.

4. K. L. Fields, On the global dimension of residue rings, Pacific J. Math. 32 (1970), 345-349. MR $42 \# 6049$.

5. A. V. Jategaonkar, Injective modules and localization in non-commutative noetherian rings, Trans. Amer. Math. Soc. 190 (1974), 109-123.

6. Y. Nouazé and P. Gabriel, Idéaux premiers de l'algèbre enveloppanté d'une algèbre de Lie nilpotente, J. Algebra 6 (1967), 77-99. MR 34 \#5889.

7. L. W. Small, A change of rings theorem, Proc. Amer. Math. Soc. 19 (1968), 662-666. MR $36 \# 6460$.

INSTITUTE OF MATHEMATICS, SNIADECKICH 8, WARSAW, POLAND 